\title{
Editorial
}

\section{Wireless Smart Sensor Networks, System, Trends, and the Applications in Engineering}

\author{
Tinggui Chen,, Gongfa Li, ${ }^{2}$ Jianjun Yang, ${ }^{3}$ and Honghai Liu ${ }^{4}$ \\ ${ }^{1}$ School of Computer and Information Engineering, Zhejiang Gongshang University, Hangzhou 310018, China \\ ${ }^{2}$ College of Machinery and Automation, Wuhan University of Science and Technology, Wuhan 430081, China \\ ${ }^{3}$ Department of Computer Science, University of North Georgia, Oakwood, GA 30566, USA \\ ${ }^{4}$ Intelligent Systems and Biomedical Robotics Group, School of Computing, University of Portsmouth, Portsmouth PO1 3HE, UK
}

Correspondence should be addressed to Tinggui Chen; ctgsimon@mail.zjgsu.edu.cn

Received 5 July 2015; Accepted 15 July 2015

Copyright (C) 2016 Tinggui Chen et al. This is an open access article distributed under the Creative Commons Attribution License, which permits unrestricted use, distribution, and reproduction in any medium, provided the original work is properly cited.

Smart environments based on Wireless Sensor Networks represent the next evolutionary development step in engineering, such as industrial automation, video surveillance, traffic monitoring, and robot control. Sensory data come from multiple networks of interconnected sensors with complex distributed locations. Wireless Sensor Networks are used in many civilian applications nowadays. On the other side, the high volume demands of human civilization and society progress have given giant feedback to wireless sensor networks.

The main objective of this special issue is to present the original research and review articles on the latest theoretical and practical achievements that will contribute to the field of Wireless Smart Sensor Networks and the applications in engineering.

The special issue received 36 high quality submissions from different countries all over the world. All submitted manuscripts have followed the same standard (peer-reviewed by at least three independent reviewers) as applied to regular ones to this journal. Inevitably, difficult decisions had to be made, and some high-quality submissions could not be included. The primary guideline was to demonstrate Wireless Smart Sensor Networks System and Trends. Besides, some novel research questions from different applications in engineering that are worthy of further investigation in the future are also included.
In the paper titled "Wireless Passive Temperature Sensor Realized on Multilayer HTCC Tapes for Harsh Environment," Q. Tan et al. design and fabricate a wireless passive temperature sensor realized on multilayer HTCC tapes. Alumina ceramic used as the substrate of the sensor is fabricated by lamination and sintering techniques, and the passive resonant circuit constituted of a planar spiral inductor and a parallel plate capacitor is printed and formed on the substrate by screen printing and postfiring processes. Since the permittivity of the ceramic becomes higher as temperature rises, the resonant frequency of the sensor decreases due to the increasing capacitance of the circuit. Measurements on the input impedance versus the resonant frequency of the sensor are achieved based on the principle, and discussions are made according to the exacted relative permittivity of the ceramic and quality factor (Q) of the sensor within the temperature range from $19^{\circ} \mathrm{C}$ (room temperature) to $900^{\circ} \mathrm{C}$. The results show the sensor demonstrates good high temperature characteristics and wide temperature range.

In the paper titled "Key Updating Methods for Combinatorial Design based Key Management Schemes," W. Liu and X. Chonghuan focus on how to update the key used in combinatorial design based key management schemes for WSNs. In order to better introduce combinatorial design, they give an example of Unital design and its mapping to key predistribution for WSNs. Then they propose two key updating methods for the Unital design based key 
management scheme, one is distributed and the other is group based. The key updating methods can be generalized into other combinatorial design based key management schemes and the group based key updating method can be modified to a self-healing version easily. They conduct the performance analysis on the two proposed methods from three aspects: storage, computation, and communication overhead. As the essence of updating keys for combinatorial design based key management schemes is the same as that of the Unital design based key management scheme, their two key updating methods can be generalized into other combinatorial design based key management schemes.

In the paper titled "Mutton Traceability Method Based on Internet of Things," Z.-X. Li et al. propose the food traceability application model, Petri network model of food traceability, and food traceability of time series data of improved $K$-means algorithm based on Internet of Things. The food traceability application model to convert, integrate, and mine the heterogeneous information, implementation of the food safety traceability information management, and Petri network model for food traceability in the process of the state transition are analyzed and simulated and provide a theoretical basis to study the behavior described in the food traceability system and structural design. The experiments on simulation data show that the proposed traceability method based on Internet of Things is more effective for mutton traceability data than the traditional $K$-means methods.

In the paper titled "A Cross-layer Wireless Sensor Network Energy-Efficient Communication Protocol for RealTime Monitoring of the Long-Distance Electric Transmission Lines," J. Yu and X. Zhang propose a linear hierarchical network topological structure specific to WSN energy conservation in environmental monitoring of the long-distance electric transmission lines in the smart grid. Based on the topological structural characteristics and optimization of network layers, the paper also proposes a Topological Structure Layered Configurations (TSLC) routing algorithm to improve the quality of WSN data transmission performance. Coprocessing of the network layer and the Media Access Control (MAC) layer is achieved by using the cross-layer design method accessing the status cross-layer of the nodes in the network layer and obtaining the status of the network nodes of the MAC layer. It efficiently saves the energy of the whole network, improves the quality of the network service performance, and prolongs the life cycle of the network.

In the paper titled "Energy Efficient Wireless Sensor Network Modelling Based on Complex Networks," X. Lin et al. consider the network topology optimization based on complex network theory to solve the energy efficiency problem of WSN. They propose the energy efficient model of WSN according to the basic principle of small world from complex networks. Small world network not only has clustering features that are similar to that of the rules of the network but also has similarity with random networks of small average path length. It can be utilized to optimize the energy efficiency of the whole network. Optimal number of multiple sink nodes of the WSN topology is given for energy efficiency. Then the hierarchical clustering analysis is used for the sensor nodes and pick up the sink nodes from the sensor nodes as the clustering head. Meanwhile, the update of the WSN when the death of certain sink node happened can cause the paralysis of network. Simulation results verify the energy efficiency of the proposed model and validate the updating of the sink nodes to ensure the normal operation of the WSN.

In the paper titled "A Distance Compensated Approach Used in Wireless Passive Pressure Sensor Readout System for High Temperature Application," Y. Hong et al. propose a distance compensated measurement system for a wireless passive sensor based on the high temperature cofired ceramics (HTCC) applied to high temperature environment. The sensor model is provided and fabricated. Additionally, a telemetric measurement system which consists of readout instrumentation and a heat insulation unit is described due to the thickness of heat insulation material between the sensor and readout unit's inductance coils in high temperature testing environment. Consideration of the leakage inductance and parasitic parameters which depend on the coupling distance is equivalent to the thickness of heat insulation material, and a distance compensated method is presented. The compensation is based on the mathematical feature of the testing results from readout unit which show us information about the relation between the extracted resonant frequencies. This method can be used simply and reliably in the other telemetric mutual inductance coupling readout system as a viable solution to compensate the coupling distance related error when inductive coupling is varied. It has been experimentally tested, and the results are in good agreement with those measured by a reference impedance analysis instrument. Theoretical explanations, experimental results, and discussion are reported.

In another paper, the research of G. Liu et al. titled "Research on an Improved Method for Permanent Magnet Synchronous Motor" proposes an improved method of PMSM control. The active disturbance rejection controller (ADRC) is designed for speed loop. Then, in order to optimize ADRC controller, the least squares support vector machines (LSSVM) optimal regression model is derived and successfully embedded in the ADRC controller. ADRC observation precision and dynamic response of the system are improved. The load disturbances' effect on the system is reduced to a large extent. The system's anti-interference ability is further improved. Finally, different sensors sampling current, voltage, and rotor speed are used to finish experimental validation.

In the paper titled "Efficient and Adaptive Node Selection for Target Tracking in Wireless Sensor Network," J. Feng et al. propose an efficient and adaptive node selection approach for tracking a target in a distributed wireless sensor network. The proposed approach combines the distancebased node selection strategy and particle filter prediction considering the spatial correlation of the different sensing nodes. Moreover, a joint distance weighted measurement is proposed to estimate the information utility of sensing nodes. Experimental results show that EANS outperformed the state-of-the-art approaches by reducing the energy cost and computational complexity and guaranteeing the tracking accuracy. 
In the paper titled "Nine Tiles Model Construction and Cache of CGML in Mobile," A. Zhang et al. put forward the construction mechanism of nine tiles model and cache organization of CGML spatial data in mobile terminals abided by nine tiles model. The model and method can monitor the map operation in mobile terminals at any moment, and they also can query and transfer map cache data ahead of time by reacting to the change of mobile map browsing. Thus mobile devices can read local CGML vector data directly in the memory for visualization, and they can accelerate the velocity of mobile cartography. This way of organization and management of mobile spatial data is good to increase the efficiency of heavy spatial data accessing in the low band and reliability of wireless network environment.

The study of Wireless Smart Sensor Networks, system, trends, and the applications in engineering is still in its early stage. This special issue demonstrates the theoretical and practical importance of further studies in it.

\section{Acknowledgments}

We would like to express our gratitude to all of the authors for their contributions and the reviewers for their effort providing valuable comments and feedback. We hope this special issue offers a comprehensive and timely view of the area of Wireless Smart Sensor Networks, system, trends, and the applications in engineering and hope it will offer stimulation for further research.

Tinggui Chen Gongfa $\mathrm{Li}$

Jianjun Yang Honghai Liu 


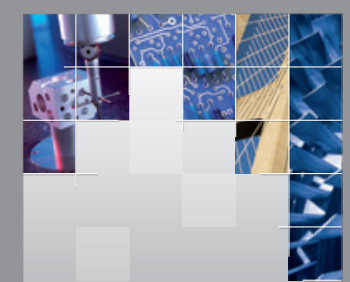

\section{Enfincering}
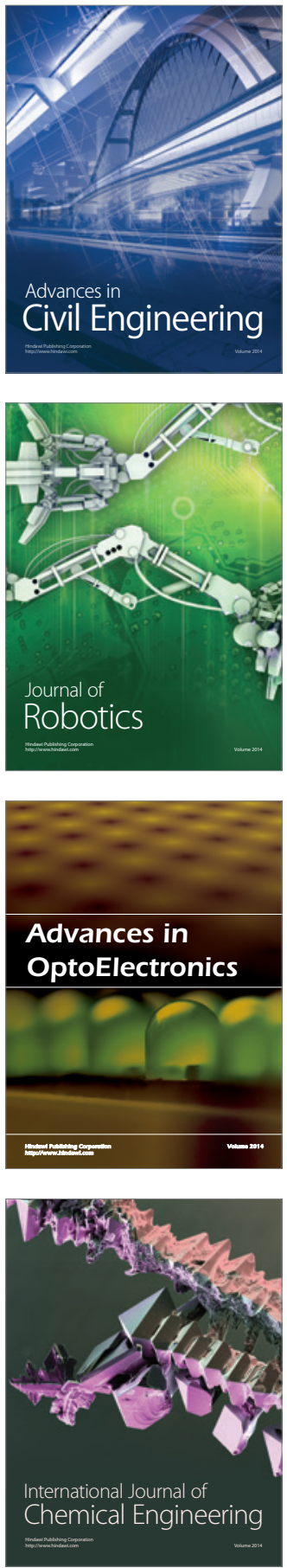

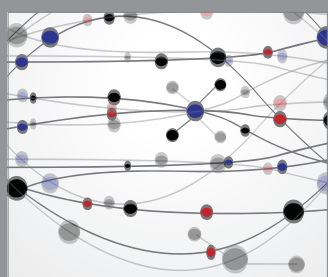

The Scientific World Journal

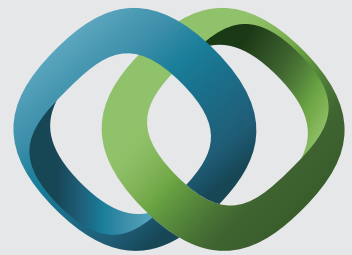

\section{Hindawi}

Submit your manuscripts at

http://www.hindawi.com
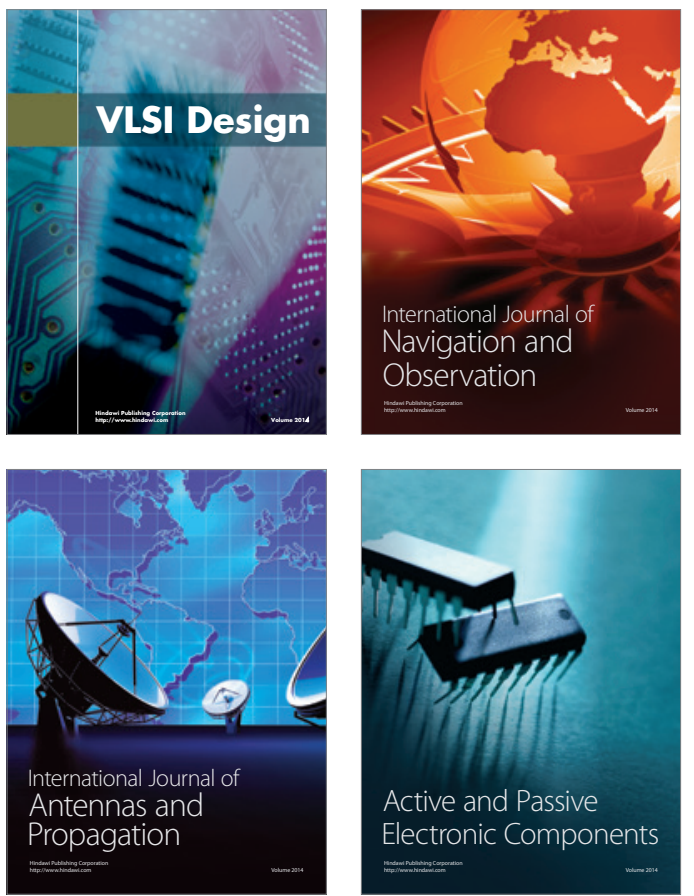
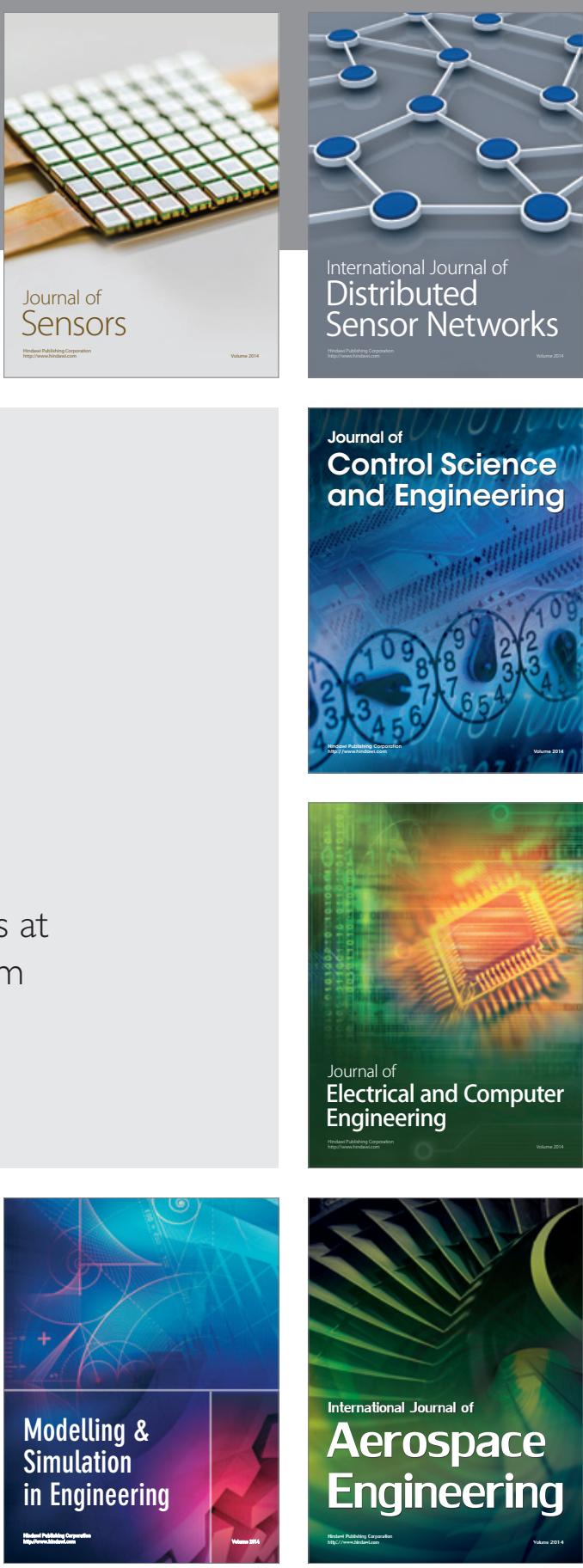

International Journal of

Distributed

Sensor Networks

Journal of

Control Science

and Engineering
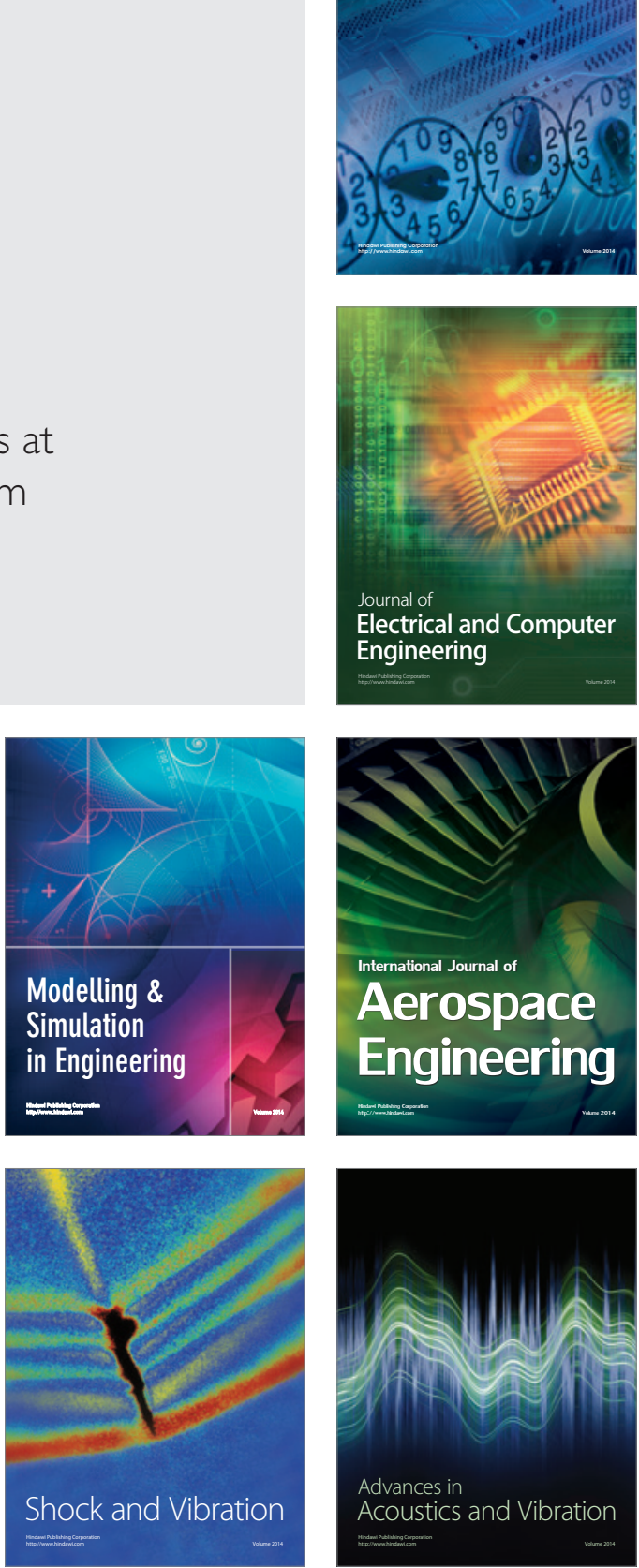